\title{
LITISPENDENCIA INTERNACIONAL Y ACTUACIONES PREVIAS AL PROCESO [CONSIDERACIONES A PROPÓSITO DE LA STJUE DE 4 DE MAYO DE 2017 (HANSEYACHTS) Y DE LA STJUE DE 20 DE DICIEMBRE DE 2017 (SCHLÖMP)]*
}

\author{
INTERNATIONAL LIS PENDENS AND PRELIMINARY \\ MEASURES [SOME REFLECTIONS IN LIGHT OF THE ECJ \\ JUDGMENTS IN THE HANSEYACHTS AND SCHLÖMP CASES]
}

\author{
Fernando Gascón InChausti \\ Catedrático de Derecho Procesal \\ Universidad Complutense de Madrid \\ Instituto de Derecho Europeo e Integración Regional (IDEIR)
}

Recibido: 10.01.2018 / Aceptado: 30.01.2018

DOI: https://doi.org/10.20318/cdt.2018.4139

\begin{abstract}
Resumen: La aplicación de las reglas sobre litispendencia internacional de las normas procesales civiles europeas se funda, de ordinario, en la prevalencia del proceso incoado en primer término. El presente trabajo aborda, a la luz de dos recientes resoluciones del Tribunal de Justicia de la Unión Europea (en los asuntos HanseYachts y Schlömp) hasta qué punto puede entenderse que generan litispendencia, a estos efectos, determinadas actuaciones previas al proceso, en especial cuando su formulación es obligatoria desde un punto de vista legal (como puede suceder con una conciliación previa) o puede resultar necesaria desde un punto de vista estratégico (como puede ser el caso de medidas de aseguramiento o anticipación probatoria o de medidas cautelares).

Palabras clave: Reglamento Bruselas I bis, Litispendencia internacional ,Comienzo del proceso, Actuaciones preprocesales, Medidas de aseguramiento y anticipación probatoria, Conciliación.
\end{abstract}

Abstract: The application of the provisions on international lis pendens established in the European regulations on civil procedure is based, ordinarily, on the prevalence of the proceeding initiated in the first place. The present work addresses, in light of two recent decisions of the Court of Justice of the European Union (in the HanseYachts and Schlömp cases) to what extent it can be understood that lis pendens, for these purposes, is produced by certain preliminary measures, prior to legal proceedings, especially when making use of them is mandatory from a legal point of view (as it can happen with a previous conciliation) or it may be necessary from a strategic point of view (this may be the case of measures to preserve or establish evidence of facts, as well as protective measures).

Keywords: Brussels I Regulation (recast), International lis pendens, Commencement of proceedings, Preliminary measures, Measures to preserve or establish evidence of facts, Conciliation.

Sumario: I. Introducción. II. La necesidad de delimitar con precisión el momento en que se entiende incoado un proceso judicial. III. Litispendencia y medidas de aseguramiento o anticipación probatoria: el asunto HanseYachts. IV. Litispendencia y conciliación previa obligatoria: el asunto Schlömp.

* El presente trabajo es uno de los resultados del Proyecto de Investigación "La armonización del proceso civil en la Unión Europea” (DER2015-64756-P), financiado por el MINECO. 


\section{Introducción}

1. La regulación de la litispendencia internacional en el ordenamiento procesal civil de la Unión Europea $^{1}$ persigue el objetivo de evitar la tramitación de procesos paralelos con el mismo objeto y de desactivar, con ello, eventuales trabas a una posterior circulación de las resoluciones judiciales. Los preceptos dedicados a esta materia han ido evolucionado en número y contenido desde el Convenio de Bruselas de 1968 hasta el Reglamento 1215/2012 (Reglamento Bruselas I bis o RB I bis, en lo sucesivo), pasando por el Reglamento 44/2001 (Reglamento Bruselas I o RB I, en lo sucesivo), con el fin de dotar al régimen jurídico de las máximas precisión y predictibilidad. No obstante, y a pesar de los cambios experimentados, el régimen normativo siempre ha descansado sobre tres postulados básicos:

2. A) La identidad de objetos procesales ("mismo objeto, misma causa, mismas partes") ha sido interpretada de forma constante por el Tribunal de Justicia de una forma amplia y flexible, que permite apreciar la concurrencia de litispendencia -y no la existencia de una mera conexión- cuando lo pretendido en ambos procesos y lo que por ende debe ser enjuiciado en ellos es "sustancialmente" lo mismo, de modo que hay identidad si es la misma relación jurídica la que se pone en tela de juicio (se trata de lo que la doctrina suele denominar Kernpunktstheorie o doctrina del núcleo esencial común). ${ }^{2}$ Esta identidad, además, es apreciable aunque uno de los dos procesos sea un proceso penal en que se está ejercitando una acción civil ex delicto, como ha tenido ocasión de recordar muy recientemente el Tribunal de Justicia. ${ }^{3}$

3. B) La existencia de una genuina situación de litispendencia internacional debe conducir en último término al sobreseimiento de uno de los dos procesos en curso -aunque ese sobreseimiento haya debido ir precedido, en un primer momento, de su paralización temporal-. ${ }^{4}$ El legislador europeo, por tanto, ha excluido el recurso a fórmulas alternativas como la acumulación de los procesos o la suspensión de uno de ellos por prejudicialidad.

4. C) Por último, la regla general para dirimir la concurrencia de procesos es la prioridad temporal, de modo que el proceso que debe prevalecer es aquel que se incoó primero: el tribunal ante el que haya comenzado el litigio en segundo lugar, una vez puesta de manifiesto la concurrencia de una situación de litispendencia internacional, debe suspenderlo de inmediato para sobreseerlo posteriormente, en

\footnotetext{
1 Para una aproximación general, sin ánimo alguno de exhaustividad, y ciñéndonos a la bibliografía en lengua española, cfr. E. Cano Bazaga, La litispendencia comunitaria, Madrid, 1997; F. Málaga Diéguez, La litispendencia, Barcelona, 1999; J.M. Chozas Alonso, "Litispendencia internacional y conexidad", en Derecho Procesal Civil Europeo. Vol. I (dir. A. de la Oliva Santos, coord. F. Gascón Inchausti), Cizur Menor, 2011, pp. 269 y ss.; M. Romero Iglesias, en Comentario al Reglamento (UE) $n^{\circ}$ 1215/2012 relativo a la competencia judicial, el reconocimiento y la ejecución de resoluciones judiciales en materia civil y mercantil (coords. P. Blanco-Morales Limones, F. Garau Sobrino, M.L. Lorenzo Guillén y F. Montero Muriel), Cizur Menor, 2016, pp. 625 y ss.; A.L. Calvo Caravaca / J. Carrascosa González, Derecho Internacional Privado, 16 ${ }^{\mathrm{a}}$ ed., Vol. I, Granada, 2016, pp. 306 y ss.; F.J. Garcimartín Alférez, Derecho Internacional Privado, 4a ed., Cizur Menor, 2017, pp. 203 y ss.

2 La vía en este dirección se abrió, como es sobradamente sabido, con la STJUE de 8 de diciembre de 1987, asunto C-144/86, Gubisch Maschinenfabrik, ECLI:EU:C:1987:528, que consideró idénticas a estos efectos una demanda pretendiendo la nulidad o la resolución de un contrato de compraventa internacional y una demanda de la parte contraria que pretende la ejecución del mismo contrato. Este criterio de identidad sustancial se ha mantenido de forma invariable con posterioridad: cfr., v.g., STJUE de 6 de diciembre de 1994, asunto C-406/92, Tatry, ECLI:EU:C:1994:400; STJUE de 19 de mayo de 1998, asunto C-351/96, Drouot assurances, ECLI:EU:C:1998:242; STJUE de 8 de mayo de 2003, asunto C-111/01, Gantner Electronic, ECLI:EU:C:2003:257; STJUE de 14 de octubre de 2004, asunto C-39/02, Moersk Olie \& Gas, ECLI:EU:C:2004:615; STJUE de 25 de octubre de 2012, asunto C-133/11, Folien Fischer y Fofitec, ECLI:EU:C:2012:664; o STJUE de 19 de diciembre de 2013, asunto C-452/12, Nipponkoa Insurance Co. (Europe) Ltd., ECLI:EU:C:2013:858. En varias de ellas, el Tribunal consideró que concurría identidad de objetos entre un proceso en que se ejercita una acción declarativa negativa de la responsabilidad de un sujeto y un proceso en que se reclama el cumplimiento de esa hipotética responsabilidad.

3 STJUE de 22 de octubre de 2015, asunto C-523/14, Aannemingsbedrijf Aertssen, ECLI:EU:C:2015:722.

4 Esta activación del mecanismo en "dos pasos" es subrayada por A.L. Calvo Caravaca / J. Carrascosa González, cit., p. 307.
} 
cuanto le conste que el tribunal del primer proceso se ha declarado competente para enjuiciarlo. ${ }^{5}$ Esta regla presenta, en el momento actual, dos excepciones:

(i) Prevalece, aunque sea más moderno, el proceso que se incoó ante el tribunal que estuviera conociendo del litigio en virtud de un fuero de competencia exclusiva, siempre, claro está, que el primer proceso se estuviera tramitando ante un tribunal de otro Estado miembro en infracción de ese fuero de competencia exclusiva; esta excepción, no prevista expresamente pero deducible del sistema, fue proclamada abiertamente por el Tribunal de Justicia en el asunto Weber. ${ }^{6}$

(ii) También prevalece desde un primer momento, aunque haya comenzado más tarde, el proceso incoado ante los tribunales designados en un pacto de sumisión expresa, con preferencia, por tanto, sobre aquél que se promovió antes, pero ante un tribunal de un Estado distinto en infracción de dicho acuerdo. Esta excepción ha sido objeto de incorporación expresa al articulado con ocasión de la refundición del RB I en el RB I bis (art. 31, aptdos. 2, 3 y 4), como reacción frente a la vigencia estricta del criterio temporal, también en este tipo de situaciones, que el Tribunal de Justicia se había visto obligado a sostener en el asunto Gasser. ${ }^{7}$ El alcance de la regla, de hecho, merece cierta precisión. No se trata, por supuesto, de que, con anterioridad a la modificación, un pacto de sumisión expresa pudiera dejarse sin efecto mediante el simple recurso de interponer la demanda en otro lugar; bajo la vigencia del régimen anterior, igual que ahora, la eficacia del pacto de sumisión expresa debía conducir al sobreseimiento del primer proceso y a la reanudación del segundo -que entre tanto solo habría quedado en suspenso-; pero, hasta entonces, las reglas generales obligaban a dejar en suspenso el segundo proceso, por ser de incoación posterior, aunque previsiblemente fuera el que habría de acabar tramitándose en cuanto el tribunal ante quien se formuló la primera demanda apreciara su ausencia de jurisdicción. ${ }^{8}$ Para evitar este "efecto torpedo", 9 el RB I bis incorporó la regla de que, en este tipo de situaciones, el proceso que debía quedar en suspenso era el incoado en infracción del pacto de sumisión expresa. ${ }^{10}$

\section{La necesidad de delimitar con precisión el momento en que se entiende incoado un proceso judicial}

5. La adecuada aplicación de un sistema de litispendencia internacional que descansa, de ordinario, en la preferencia temporal requiere que resulte sencillo determinar el momento en que se entiende incoado un proceso judicial. Sin perjuicio de una eventual aplicación de oficio de las reglas del RB I bis, lo habitual será que el demandado en el segundo proceso suscite la cuestión al tribunal que conoce de ese segundo proceso y le solicite por de pronto la suspensión, como paso previo para un posterior sobreseimiento. El tribunal del segundo proceso, por ello, se verá obligado a determinar si, como sostiene

5 Cuándo debe considerarse que el tribunal del primer proceso se ha declarado competente es algo sobre lo que el Tribunal de Justicia se pronunció en su Sentencia de 27 de febrero de 2014, asunto C-1/13, Cartier parfums - lunettes SAS, ECLI:EU:C:2014:109, en los siguientes términos: “(...) deberá considerarse que el tribunal ante el que se presentó la primera demanda se ha declarado competente, a efectos de la citada disposición, cuando dicho tribunal no haya declinado de oficio su competencia y ninguna de las partes haya impugnado tal competencia con anterioridad o en el momento de la actuación procesal que el Derecho procesal nacional considere como el primer medio de defensa sobre el fondo invocado ante ese tribunal."

6 STJUE de 3 de abril de 2014, asunto C-438/12, Weber, ECLI:EU:C:2014:212.

7 STJUE de 9 de diciembre de 2003, asunto C-116/02, Gasser, ECLI:EU:C:2003:657. Cfr. F.J. Garcimartín Alférez / S. SÁnCHEZ FERnÁNDEZ, "El nuevo Reglamento Bruselas I: qué ha cambiado en el ámbito de la competencia judicial", Revista española de derecho europeo, №. 48, 2013, pp.. 9-35; F.J. GARCimartín Alférez, en The Brussels I Regulation Recast (eds. A. Dickinson / E. Lein), OUP, 2015, pp. 336-343.

8 STJUE de 27 de junio de 1991, asunto C-351/89, Overseas Union Insurance, ECLI:EU:C:1991:279.

9 Cfr. A.L. Calvo Caravaca / J. Carrascosa González, cit., pp. 308-311.

10 Cfr., no obstante, las apreciaciones de J. Suderow, "Nuevas normas de litispendencia y conexidad para Europa: ¿el ocaso del torpedo italiano? ¿flexibilidad versus previsibilidad?", Cuadernos de Derecho Transnacional, Vol. 5, No. 1, 2013, pp. 184-198. 
la parte demandada, el litigio que ante él se sustancia fue incoado con posterioridad al otro y, para ello, habrá de establecer el momento exacto en que comenzó cada uno de ellos.

6. Esta operación sería sencilla si los ordenamientos procesales nacionales fuesen iguales en este punto o si, al menos, hubieran sido armonizados. Lo cierto, sin embargo, es que las reglas sobre incoación del proceso suelen ser diferentes, pues se hallan estrechamente ligadas a la tradición forense y a la cultura procesal de cada Estado miembro: en algunos países, v.g., la demanda se interpone directamente ante el tribunal competente, siendo después necesaria su notificación al demandado, que en ciertos sistemas se hace de oficio por el tribunal, mientras que en otros es el demandante quien ha de encargarse de llevarla a cabo, normalmente a través de determinados profesionales jurídicos; pero también existen ordenamientos en los que debe haberse notificado la demanda previamente al demandado antes de presentarla al tribunal. ${ }^{11}$

7. Resulta, pues, que una situación de concurrencia entre procesos debe ser resuelta sobre la base de la decisión que tome uno de los tribunales en liza quien, además, se ve en la necesidad de aplicar una normativa procesal que no es la suya para definir cuál de los dos litigios comenzó antes. Y, a menudo, debe hacerlo en situaciones de "carrera judicial" (race to courts o rush to courts), es decir en casos en los que las partes enfrentadas en una controversia compiten por formular la primera demanda y asegurarse así la litigación en un terreno que consideran más propicio y cómodo para sí o, al menos, más incómodo para el adversario.

8. Durante el periodo inicial de vigencia del CB (esto es, hasta que fue reemplazado en 2001 por el RB I) no había en el régimen normativo europeo ninguna regla especial al respecto, de modo que no quedaba más remedio que remitirse al sistema nacional de cada Estado miembro. El riesgo de este silencio, por supuesto, consistía en que el tribunal del segundo proceso podría tender a utilizar sus propias reglas para determinar el momento en que cabía entender que había comenzado el otro proceso. Se daba pábulo, con ello, a que se suscitaran problemas de comprensión recíproca y a disfunciones en la puesta en marcha del sistema, que fueron puestos de manifiesto por el Tribunal de Justicia en el asunto Zelger. ${ }^{12}$

9. El legislador europeo trató de remediar esta dificultad con ocasión de la elaboración del RB I y la introducción en él de un nuevo precepto, que se ha mantenido en el RB I bis como artículo 32 y que tiene la finalidad de definir de manera autónoma ${ }^{13}$ cuándo se entiende incoado un litigio, agrupando los sistemas procesales de los Estados miembros en dos grandes categorías, en función del momento en que se entiende que un proceso ha comenzado, y ello a su vez en función del modo en que se reparten funciones el demandante y el tribunal a la hora de poner la existencia del proceso en conocimiento del demandado.

${ }^{11}$ Cfr. al respecto el estudio comparado que se ofrece en An evaluation study of national procedural laws and practices in terms of their impact on the free circulation of judgments and on the equivalence and effectiveness of the procedural protection of consumers under EU consumer law. Strand 1: mutual trust and free circulation of judgments, elaborado desde el Max Planck Institute Luxembourg for Procedural Law bajo la dirección de B. Hess y accessible en https://publications.europa.eu/en/publication-detail/-/publication/531ef49a-9768-11e7-b92d-01aa75ed71a1/language-en

12 STJUE de 7 de junio de 1984, asunto C-129/83, Zelger, ECLI:EU:C:1984:215: "El artículo 21 del Convenio de 27 de septiembre de 1968 relativo a la competencia judicial y a la ejecución de resoluciones judiciales en materia civil y mercantil debe interpretarse en el sentido de que "el Tribunal ante el que se formule la primera demanda" es aquel ante el cual han concurrido en primer lugar los requisitos para que se produzca una litispendencia definitiva, debiendo apreciarse estos requisitos según la ley nacional de cada uno de los órganos jurisdiccionales afectados”.

13 Esa autonomía significa, en la práctica, que se ha de considerar incoado un proceso a efectos de aplicar las reglas procesales civiles europeas aunque, con arreglo a la normativa procesal nacional, el proceso aún no se debiera considerar iniciado. Aunque sea paradójico, así lo ha proclamado expresamente el Tribunal de Justicia en el asunto $M$. $H$. respecto del Reglamento 2201/2003 en materia matrimonial y de responsabilidad parental [ATJUE de 22 de junio de 2016, asunto C173/16, M.H., ECLI:EU:C:2016:542: "El artículo 16, apartado 1, letra a), del Reglamento (CE) n. ${ }^{\circ}$ 2201/2003 del Consejo, de 27 de noviembre de 2003, relativo a la competencia, el reconocimiento y la ejecución de resoluciones judiciales en materia matrimonial y de responsabilidad parental, por el que se deroga el Reglamento (CE) n. ${ }^{\circ} 1347 / 2000$, debe interpretarse en el sentido de que el "momento en que se [presenta ante un órgano jurisdiccional] el escrito de demanda o documento equivalente", con arreglo a dicha disposición, es el momento en que dicha presentación tiene lugar ante el órgano judicial de que se trate, aun cuando esa presentación no inicie por sí misma, de manera inmediata, el procedimiento según el Derecho nacional’”. 
"Artículo 32

1. A efectos de la presente sección, se considerará que un órgano jurisdiccional conoce de un litigio:

a) desde el momento en que se le presente el escrito de demanda o documento equivalente, a condición de que posteriormente el demandante no deje de tomar todas las medidas que se le exijan para que se entregue al demandado la cédula de emplazamiento, o

b) si dicho documento ha de notificarse al demandado antes de su presentación al órgano jurisdiccional, en el momento en que lo reciba la autoridad encargada de la notificación, a condición de que posteriormente el demandante no deje de tomar todas las medidas que se le exijan para presentar el documento al órgano jurisdiccional.

La autoridad encargada de la notificación a que se refiere la letra b) será la primera autoridad que reciba los documentos que deban notificarse.

2. Los órganos jurisdiccionales, o las autoridades encargadas de la notificación, a que se refiere el apartado 1, consignarán, respectivamente, la fecha de presentación del escrito de demanda o documento equivalente o la fecha de recepción de los documentos que deban notificarse."

10. Se opta, pues, por un sistema que trata de premiar la diligencia del demandante y que evita que pueda verse perjudicado por eventuales dilaciones atribuibles a terceros (tribunal incluido) que se encuentren fuera de su esfera de control. ${ }^{14}$ Con estos parámetros y con una alegación y acreditación adecuada del ordenamiento procesal extranjero por parte del demandado que le pide la suspensión y el sobreseimiento, el tribunal del segundo proceso debería estar en condiciones de adoptar una decisión que fuera respetuosa con el esquema del Reglamento.

11. Las dudas, sin embargo, no terminan con un precepto que establezca de forma autónoma la fecha en que se entiende incoado un proceso sobre el fondo. Puede haber problemas, v.g., en caso de que haya varios demandados y la notificación a cada uno se haya hecho en fechas diversas o cuando la demanda es objeto de ampliación con posterioridad. ${ }^{15}$ Además, debe tenerse en cuenta que el tenor literal del artículo 32 RB I bis parte de la premisa implícita de que están compitiendo dos procesos civiles "clásicos" u "ordinarios". Ahora bien, no tiene por qué ser siempre así. Uno de los procesos en liza, como ya se apuntó antes, puede ser un proceso penal en que se esté ejercitando una acción civil ex delicto. En tal caso, a juicio del Tribunal de Justicia, debe interpretarse que la demanda ha sido formulada cuando se ha presentado una denuncia con personación como actor civil ante un juez de instrucción, aunque no se haya completado todavía la fase de instrucción del asunto controvertido. ${ }^{16}$

12. $Y$, en especial, es frecuente que con carácter previo a la interposición de una demanda "clásica" sobre el fondo del litigio, el demandante desarrolle - o deba desarrollar- determinadas actuaciones previas, de cuyo desenlace dependerá la posterior incoación formal de un proceso sobre el fondo. Se hace necesario, en consecuencia, determinar si dichas actuaciones "generan litispendencia internacional" y otorgan con ello prioridad temporal a los tribunales del Estado en que se desarrollaron, si con posterioridad se interpone una demanda sobre el mismo objeto ante un tribunal de un Estado diferente. El Tribunal de Justicia, de hecho, se ha enfrentado en fechas recientes a dos situaciones diversas susceptibles de encaje en estos parámetros: la solicitud de medidas de anticipación probatoria con carácter previo a la demanda (asunto HanseYachts) y el desarrollo de una conciliación obligatoria previa a la demanda (asunto Schlömp).

\section{Litispendencia y medidas de aseguramiento o anticipación probatoria: el asunto HanseYachts}

13. En el asunto HanseYachts ${ }^{17}$ se suscitó la cuestión de si, a los efectos de la aplicación de las reglas de litispendencia internacional, podía considerarse que un proceso civil comienza cuando se

\footnotetext{
${ }^{14}$ En este sentido cfr. también J.M. Chozas Alonso, cit., pp. 286-287.

15 Cfr. P. Rogerson, en The Brussels I Regulation Recast (eds. A. Dickinson / E. Lein), OUP, 2015, pp. 344-345.

${ }^{16}$ Cfr. STJUE de 22 de octubre de 2015, asunto C-523/14, Aannemingsbedrijf Aertssen, ECLI:EU:C:2015:722.

17 STJUE de 4 de mayo de 2017, asunto C-29/16, HanseYachts AG, ECLI:EU:C:2017:343.
} 
solicita del tribunal la adopción de medidas para el aseguramiento o la práctica de pruebas con carácter previo a la interposición de una demanda en cuanto al fondo -todo ello, al amparo de lo previsto en el artículo 145 del Code de procédure civile francés, que regula las llamadas mesures d'instruction in futurum, a veces también référé probatoire ${ }^{18}$.

14. La secuencia de los hechos del caso se puede imaginar con facilidad: en agosto de 2011, el comprador de una embarcación a motor, a raíz de una avería sobrevenida, solicitó al tribunal de commerce de Marsella que, al amparo del artículo 145 del CPC francés, ordenase que se llevara a cabo un peritaje judicial previo a cualquier proceso sobre el fondo. Entre los demandados en el procedimiento de medidas provisionales se hallaba la empresa constructora de la embarcación, la sociedad alemana HanseYachts. El perito designado por el tribunal francés emitió su informe tres años después, en septiembre de 2014. En noviembre de 2014, la constructora HanseYachts interpuso una demanda declarativa negativa ante el Landgericht de Stralsund (Alemania), solicitando que se declarase su ausencia de responsabilidad frente a cualquiera de los demás sujetos involucrados (adquirente final, vendedor y aseguradora del vendedor). En enero de 2015, por último, la compradora interpuso ante el tribunal de commerce de Toulon demanda frente al vendedor, el fabricante del motor y HanseYachts (respecto de esta última, sobre la base de su deber de garantía como fabricante por vicios ocultos), solicitando la reparación del perjuicio, así como el reembolso de los gastos provocados por el peritaje judicial.

15. Ante el Landgericht de Stralsund las entidades demandadas por HanseYachts formularon la correspondiente excepción de litispendencia internacional, por considerar que las actuaciones judiciales ante los tribunales franceses debían ser consideradas como integrantes de un único proceso, que a su vez debía entenderse comenzado en agosto de 2011, cuando se solicitó el peritaje judicial ante demandam, y no en enero de 2015, cuando se interpuso la demanda en cuanto al fondo: eso determinaría que el proceso ante el tribunal alemán fuera, a estos efectos, el "segundo" proceso, al que debería ponerse fin de forma anticipada. Las dudas del Landgericht determinan el planteamiento por su parte de la correspondiente cuestión prejudicial ante el Tribunal de Justicia.

16. Desde el punto de vista formal, la cuestión prejudicial se le presenta al Tribunal de manera parcialmente diversa: se trata de determinar si una solicitud de medidas de anticipación probatoria -al amparo, en este caso, de la normativa procesal civil francesa- puede considerarse como "documento equivalente" a un "escrito de demanda", a los efectos de aplicar las reglas de determinación del momento de incoación del proceso del artículo $32 \mathrm{RB}$ I bis (art. $30 \mathrm{RB}$ I, aplicable al caso por razones temporales). Sea cual sea el enfoque, el problema es el mismo: la incidencia de una actuación preprocesal sobre el momento de comienzo de la litispendencia.

17. $Y$ es que, por una parte, resulta evidente que el proceso sobre el fondo no comienza en sentido propio cuando se solicita una medida de aseguramiento y/o anticipación probatoria, como la que nos ocupa en el presente asunto. No resulta fácil hablar de litispendencia, por ello, cuando no es seguro, siquiera, si llegará a haber proceso ni se sabe, en su caso, cuál será su objeto (qué se pedirá, frente a quién y por qué razones): esto depende, justamente, del desenlace de la medida en cuestión.

18. Pero, por otro lado, tampoco cabe ignorar que en determinadas situaciones, la interposición de una demanda que pueda tener visos de prosperar requiere de la realización de actuaciones previas,

\footnotetext{
18 "S'il existe un motif légitime de conserver ou d'établir avant tout procès la preuve de faits dont pourrait dépendre la solution d'un litige, les mesures d'instruction légalement admissibles peuvent être ordonnées à la demande de tout intéressé, sur requête ou en référé". La traducción al castellano del precepto que contiene la sentencia es esta: " "Si existe un motivo legítimo para asegurar o constituir, antes de cualquier proceso, pruebas de los hechos de los que pudiera depender la resolución de un litigio, podrán ordenarse las diligencias de prueba legalmente admisibles a petición de cualquier interesado, mediante demanda o en el marco de un procedimiento de medidas provisionales."

Sobre esta figura, cfr. entre otros L. Cadiet / E. Jeuland, Droit judiciaire privé, pp. 465-468 $8^{\mathrm{a}}$ ed., París, 2013; C. Chainais / F. Ferrand / S. Guinchard, Procédure civile, 33ª ed., París, 2016, pp. 1319-1326; E. Vergès / G. Vial / O. Leclerc, Droit de la preuve, París, 2015, pp. 309-312.
} 
para las que en algunos casos puede ser necesario o conveniente contar con la colaboración de un tribunal (v.g., para vencer resistencias de la contraparte o de terceros). En estos casos, desde un punto de vista funcional, se podría entender que para el demandante diligente la actividad procesal encaminada a obtener la tutela y realización de su derecho comienza cuando formula su solicitud al tribunal -en este caso, su solicitud de anticipación y aseguramiento probatorio-.

19. El TJUE resuelve la cuestión en sentido negativo: “(...) en caso de litispendencia, la fecha en la que se ha iniciado un procedimiento dirigido a obtener una diligencia de prueba antes de cualquier proceso no puede constituir la fecha en la que "se considerará que conoce de un litigio", en el sentido del referido artículo 30, punto 1 , un órgano jurisdiccional que ha de pronunciarse sobre una demanda en cuanto al fondo presentada en ese mismo Estado miembro con posterioridad al resultado de dicha diligencia". Para sostener su criterio el Tribunal, sin embargo, no entra en apreciaciones como las señaladas más arriba, sino que su decisión se funda como argumento básico en la autonomía entre el proceso de aseguramiento probatorio y el proceso declarativo en cuanto al fondo, refrendada por el gobierno francés en sus alegaciones: la legislación y la praxis judicial francesas reconocen que las mesures d'instruction in futurum tienen autonomía procesal y no pueden considerarse como parte o prolegómeno formal del proceso sobre el fondo. Siendo así, difícilmente puede sostenerse que, una vez concluido el procedimiento probatorio, se genere una especie de ficción que lo ligue con un proceso declarativo incoado meses después y, además, ante un tribunal distinto. Estos dos últimos factores, de hecho, sirven para apuntalar el dato formal de la autonomía procedimental y para despejar cualquier posible duda que hubiera podido albergar el Tribunal de Justicia.

20. La dualidad de tribunales, en efecto, puede tomarse como un síntoma de independencia procesal, aunque no es en absoluto determinante: no es nada extraño que la competencia para adoptar actuaciones preprocesales se rija por criterios distintos a los ordinarios para conocer del fondo del asunto, pues suelen primar factores que permitan una mayor cercanía del tribunal con el objeto de las medidas o que aseguren una mayor rapidez para su puesta en práctica. Esta divergencia de tribunales, por sí sola, no debería considerarse un elemento determinante para resolver una cuestión como la suscitada.

21. El segundo factor, en cambio, sí que puede tener mayor fuerza de convicción: la secuencia temporal de acontecimientos pone de relieve que la compradora de la embarcación no fue singularmente veloz a la hora de interponer su demanda, pues esperó casi cinco meses para hacerlo desde la conclusión del peritaje judicial. No se trata sólo de que exista una clara desconexión entre las mesures d'instruction in futurum del artículo 145 CPC francés y el posterior proceso sobre el fondo; es que, además, la relativa pasividad de la compradora de la embarcación puso de manifiesto una aparente falta de diligencia por su parte en la defensa activa de su posición jurídica. Y, según se ha apuntado antes, el criterio relevante que subyace a la opción del legislador europeo para determinar la prioridad temporal es justamente el de no perjudicar al demandante activo y diligente. Cabe añadir, además, un factor que no figura en la sentencia, pero que tampoco es desdeñable: la propietaria de la embarcación podría haber efectuado el peritaje a título particular, sin necesidad de acudir a los tribunales, puesto que aquella se hallaba en su poder y no le era preciso vencer eventuales resistencias para llevarla a cabo; en otros términos, el recurso a las mesures d'instruction in futurum, en el caso concreto, fue más una cuestión de preferencia o conveniencia que de necesidad.

22. Por todo ello, cabe plantearse si la respuesta del Tribunal de Justicia habría sido la misma en caso de que la embarcación sobre la que practicar el peritaje se hallara en posesión de otro sujeto y la empresa fabricante HanseYacths hubiera interpuesto su acción declarativa negativa en Alemania antes incluso de que el perito hubiera presentado su informe, o tan solo unos pocos días después, pero igualmente pocos días antes de la formulación en Francia de la demanda en cuanto al fondo por parte de la compradora. No tengo tan claro que, con este cambio de variables, la argumentación del Tribunal hubiera podido mantenerse: la medida habría sido considerada necesaria -si no legalmente, sí desde un punto de vista estratégico- y la diligencia del actor habría sido igualmente incuestionable. 
23. Y es que la respuesta del TJUE, en el caso concreto que se le planteó, resulta razonable dadas las circunstancias del litigio y dada la función de las mesures d'instruction in futurum en el contexto del proceso civil francés. Ahora bien, creo que ni los argumentos ni las respuestas resultan generalizables a cualquier tipo de actividad preprocesal de carácter preparatorio o asegurativo, conforme a cualquier ordenamiento procesal nacional: en otros términos, no puede decirse que se trate de una duda ya zanjada en la jurisprudencia del Tribunal de Justicia. ${ }^{19}$ Problemas similares pueden suscitarse, por ejemplo, cuando se trate de medidas cautelares ante demandam, tanto en modelos procesales en que la instrumentalidad de las medidas cautelares es rígida como en otros en que sea más flexible. Y la duda se podrá plantear también cuando se soliciten diligencias preliminares o cuando se formulen peticiones de acceso a fuentes de prueba (v.g., al amparo de los instrumentos que han desarrollado la Directiva 2004/48 -tutela judicial de los derechos de propiedad intelectual e industrial- o la Directiva 2014/104 -acciones de daños en materia de defensa de la competencia-).

24. Cuando se solicitan actuaciones preprocesales dirigidas a preparar el litigio o asegurar su eficacia no se está entablando aún una acción en cuanto al fondo: eso es algo evidente. Ahora bien, en determinadas situaciones el litigante que aspira a defender de la forma más diligente su posición jurídica se ve constreñido a solicitar medidas previas a la demanda, sea para conseguir información o pruebas necesarias en poder del adversario o de terceros, sea para paliar o mitigar un periculum in mora acreditable: si no lo hace corre el riesgo de incurrir en fallos estratégicos que pueden ser irreversibles. Y, de hecho, para que este tipo de medidas prosperen suele ser imprescindible que el solicitante identifique con precisión cuál habrá de ser el objeto del proceso que se propone someter a enjuiciamiento en el futuro. Sería paradójico que, en semejante tesitura, mientras se están tramitando estas actuaciones a instancia de uno de los litigantes en un Estado, pudiera la parte contraria acudir a los tribunales de otro Estado miembro e interponer una demanda ejercitando una acción declarativa negativa $-\mathrm{o}$ de alcance práctico equivalente-, lograr la prioridad temporal a efectos de litispendencia internacional y condenar con ello al sobreseimiento a cualquier eventual proceso en cuanto al fondo que hubiera de ser subsiguiente a las actuaciones preprocesales en curso $-\mathrm{y}$, de paso, a las medidas en sí mismas, pues carecería de sentido preparar o asegurar la eficacia de un proceso que no podría tramitarse-.

25. En definitiva, una generalización extensiva de la respuesta ofrecida por el TJUE en el asunto HanseYachts abriría un margen para el abuso y para la formulación de demandas torpedo que tampoco parece compatible con la finalidad del Reglamento. A la inversa, por supuesto, sería igualmente disfuncional que la sola solicitud de una medida cautelar, una medida de aseguramiento o anticipación probatoria, una diligencia preliminar o una medida de acceso a fuentes de prueba pudiera usarse de forma fraudulenta para obtener sin más la prioridad temporal a efectos de resolver una situación de litispendencia internacional en un contexto de race to the courts transfronterizo. Para medir esta variable pueden ser de utilidad las normas procesales internas que supeditan la eficacia de las actuaciones preprocesales a la interposición de la demanda en un plazo determinado: ese es el lapso temporal que se considera razonable para levantar diligentemente la carga de formular demanda una vez se ha obtenido una medida cautelar, una diligencia preliminar, el aseguramiento de una fuente de prueba, la práctica de prueba anticipada o el acceso a una fuente probatoria.

26. Las soluciones generales, por tanto, no existen, sino que en cada caso concreto será preciso ponderar las variables concurrentes para determinar a quién corresponde la preferencia temporal en un contexto de litispendencia internacional $-\mathrm{y}$, posiblemente, también en supuestos meramente internos, en que estos problemas son igual de imaginables-. Entre esas variables que han de ponderarse el mayor peso debe otorgarse, a mi juicio, al grado de necesidad o constreñimiento experimentado por quien solicita la medida previa al proceso, atendidas las circunstancias del caso: este dato es el más expresivo de la diligencia del demandante, que fundamenta a su vez la asignación de prioridad temporal. Junto a ello, por supuesto, habrá que tener en cuenta el grado de instrumentalidad existente entre la medida

19 Cfr., no obstante, Garcimartín AlfÉrez, cit., p. 207. 
previa y el posterior desarrollo del proceso en cuanto al fondo, así como el nivel de diligencia mostrado en concreto (v.g., en relación con los tiempos y los plazos) por cada uno de los protagonistas del litigio en la defensa de su posición jurídica.

27. Es cierto que ello impide hacer afirmaciones generales que doten al sistema del nivel de predictibilidad que sería deseable; y existe igualmente el riesgo de que ponderaciones diferentes a cada lado de la frontera pueden conducir a situaciones reales de doble pendencia, por considerar ambos tribunales que las actuaciones procesales desarrolladas ante ellos son las que han de entenderse incoadas en primer lugar. Se trata, no obstante, de la única manera de resolver el problema sin colocar a los litigantes ante genuinos callejones sin salida.

\section{Litispendencia y conciliación previa obligatoria: el asunto Schlömp}

28. Las dudas y los problemas apuntados se reproducen con ocasión de las conciliaciones previas obligatorias, que cada vez con mayor frecuencia se imponen por las legislaciones procesales nacionales como requisito para la admisibilidad posterior de una demanda. ${ }^{20}$ Esta fue la cuestión resuelta en fecha muy reciente por el Tribunal de Justicia en el asunto Schlömp, ${ }^{21}$ en relación con el ordenamiento procesal suizo y bajo el prisma del Convenio de Lugano II, que en este punto no presenta diferencias ni con el RB I ni con el RB I bis.

29. El 16 de octubre de 2015 una entidad pública alemana interpuso solicitud de conciliación frente a la Sra. Schlömp ante el juez de paz del distrito de Reiat (Suiza), reclamándole el reembolso de una cantidad de dinero adelantada por dicha entidad para hacer frente a las necesidades asociadas a la dependencia de su madre, internada en una residencia de Alemania: con arreglo al ordenamiento procesal civil suizo, este intento de conciliación es obligatorio para un eventual y posterior ejercicio judicial de acciones. El intento de conciliación resultó infructuoso, de modo que el 25 de enero de 2016 el juez de paz autorizó a la entidad pública alemana a formular demanda, algo que hizo ante el Kantonsgericht de Schaffhausen (Suiza) el 11 de mayo de 2016. No obstante, el 19 de febrero de 2016 la Sra. Schlömp ya había interpuesto ante el Amtsgericht de Schwäbisch Hall (Alemania) una demanda declarativa negativa, que fue posteriormente reenviada al Amtsgericht de Stuttgart, ante el que la entidad pública alemana formuló la excepción de litispendencia internacional. Fue la cuestión prejudicial formulada por este órgano judicial la que dio lugar al pronunciamiento del Tribunal de Justicia. En efecto, si se tiene en cuenta únicamente la fecha de incoación de los dos procesos judiciales, tendría preferencia el incoado ante el tribunal alemán. Sin embargo, si se considera que la fase inicial de conciliación, aún sin ser proceso en sentido propio, genera litispendencia, la solución sería la contraria y deberían proseguir las actuaciones ante el tribunal suizo.

30. Se trataba, de nuevo, pues, de determinar si una actuación preprocesal es o no susceptible de generar una situación de litispendencia o, más propiamente, de determinar la fecha de incoación del proceso a efectos de determinar la preferencia temporal. Y, también aquí como en el asunto HanseYachts, el enfoque de la cuestión prejudicial ante el TJUE fue parcialmente indirecto: no se planteaba sólo si la fecha en que se formuló la solicitud de conciliación servía también como fecha de incoación del proceso en cuanto al fondo, sino que se añadió la variable de la naturaleza jurídica del órgano de conciliación,

${ }^{20}$ Y recuérdese que el Tribunal de Justicia ha considerado que la imposición de un procedimiento de conciliación o mediación con carácter previo a la formulación de una demanda no resulta incompatible con el derecho a la tutela judicial efectiva consagrado en el artículo 47 de la Carta de los Derechos Fundamentales de la Unión Europea [cfr. en este sentido STJUE de 18 de marzo de 2010, asunto C-317/08, Rosalba Alassini, ECLI:EU:C:2010:146; y STJUE de 14 de junio de 2017, asunto C-75/16, Menini y Rampanelli, ECLI:EU:C:2017:457]. Tampoco lo es para el Tribunal Europeo de Derechos Humanos, desde el prisma del derecho de acceso a un tribunal al amparo del artículo 6.1 del Convenio Europeo de Derechos Humanos: cfr. STEDH de 26 de marzo de 2015, asunto 11239/11, Momćilović c. Croacia.

${ }^{21}$ Cfr. STJUE de 20 de diciembre de 2017, asunto C-467/16, Schlömp, ECLI:EU:C:2017:993. 
esto es, si cabe reconocerlo como "tribunal" a los efectos de aplicar las reglas del Convenio de Lugano: no era, pues, solo una cuestión de fechas, sino que estaba también en juego el carácter no jurisdiccional stricto sensu del procedimiento de conciliación.

31. La respuesta del Tribunal fue clara y directa: "en caso de litispendencia, la fecha en que se inicie un procedimiento obligatorio de conciliación ante un órgano de conciliación de Derecho suizo es la fecha en la que se considera que un "tribunal" conoce del litigio".

32. La solución -también aquí- vino facilitada por los datos positivos en relación con la legislación procesal suiza. De un lado, en el Código procesal civil suizo existe un precepto, el artículo 62.1, que de manera expresa establece a efectos internos que la litispendencia se produce desde que se presenta la solicitud de conciliación, la demanda o la demanda de divorcio de mutuo acuerdo. En términos equivalentes, la Ley federal sobre el derecho internacional privado, al abordar la litispendencia internacional, dispone en su artículo 9.2 que "[p]ara determinar el momento en que una acción está pendiente en Suiza, será determinante la fecha de la primera actuación procesal necesaria para iniciar la instancia. La citación para conciliación será suficiente". Aunque ambos extremos no son per se suficientes para interpretar la normativa europea (en este caso, el Convenio de Lugano), dado el carácter autónomo de esta última, sí que son claramente expresivos de lo que, a mi juicio, es el núcleo del problema y la clave para su solución: el carácter obligatorio de la actuación preprocesal.

33. Si la conciliación es obligatoria el litigante diligente no puede evitarla, razón por la cual debe entenderse que su tramitación forma parte del proceso a efectos de litispendencia internacional: con arreglo al Derecho suizo, formular una solicitud de conciliación supone emprender el primer paso para la defensa de su posición jurídica. Además, el ordenamiento suizo señala expresamente que la solicitud de conciliación debe contener una identificación del objeto del litigio (art. 202.2 CPC suizo), de modo que existe un parámetro suficiente para comparar su objeto con el de cualquier otro proceso, nacional o extranjero. Obviamente, esta eficacia de la solicitud de conciliación no puede mantenerse de forma indefinida: también para esto existe previsión expresa, que otorga al demandante un plazo de tres meses para interponer la demanda desde que se le conceda la correspondiente autorización, tras constatar el fracaso de la conciliación (art. 209.3 CPC). Las reglas del Derecho suizo, por tanto, ofrecen una ponderación adecuada entre las exigencias de seguridad jurídica y el reconocimiento de los esfuerzos del litigante diligente para la defensa de su posición jurídica.

34. En cuanto al segundo posible escollo -si los órganos de conciliación suizos son o no "tribunales" a los efectos de poder aplicar las reglas de litispendencia internacional del Convenio de Lugano-, el Tribunal de Justicia lo despeja de forma expeditiva, nuevamente con la comodidad que le proporciona la legislación nacional suiza, con arreglo a la cual los órganos de conciliación pueden ser recusados con arreglo a las mismas normas que los jueces de paz que forman parte de ellos y, además, ejercen sus funciones con total autonomía. Estos dos datos -imparcialidad y autonomía- han de sumarse a la noción propia de "tribunal" que maneja la legislación procesal civil europea, de carácter estrictamente funcional, al margen de las categorías propias de los Derechos nacionales. Y, de hecho, podrían extrapolarse para resolver situaciones similares a la planteada en el caso Schlömp, cuando la conciliación o la mediación previas y obligatorias, en vez de atribuirse a órganos integrados en las estructuras del poder judicial -como los juzgados de paz suizos- o cercanos a ellas, estén encomendadas a entidades u organismos administrativos, si se aseguran la imparcialidad y la autonomía funcional de aquellas y de las concretas personas que ejerzan funciones de conciliador o de mediador.

35. La solución ofrecida por el Tribunal de Justicia es así susceptible de generalización, con independencia de lo que pueda establecer en concreto la legislación procesal nacional: si un litigante se ve forzado a acudir a un procedimiento de solución extrajudicial de controversias antes de poder formular una demanda judicial, debe entenderse, a efectos de litispendencia internacional, que el proceso comienza en el momento en que se formula la correspondiente solicitud. No sólo se atiende con ello 
al fundamento de las reglas sobre asignación de preferencia temporal de las normas procesales civiles europeas, sino que también se evita el callejón sin salida al que conduciría la obligación, impuesta por determinadas legislaciones nacionales -en general o para ciertas materias-, de acudir a mediaciones o conciliaciones previas a todo proceso, si el lapso de tiempo necesario para desarrollarlas hasta constatar su fracaso pudiera ser aprovechado por la contraparte para presentar una demanda torpedo ante los tribunales de un Estado que no impusiera semejante deber. 